\title{
Eficacia de la budesonida/formoterol comparado con la fluticasona/salmeterol en la mejoría de la capacidad pulmonar de personas mayores con Enfermedad Pulmonar Obstructiva Crónica: revisión sistemática de literatura
}

\author{
Ledmar J Vargas-Rodríguez ${ }^{1}$, Flore E Solano-Rojas², Sandra L Díaz ${ }^{3}$, Manuel A Pinzón-Olmos ${ }^{4}$
}

\begin{abstract}
RESUMEN
Introducción. La Enfermedad Pulmonar Obstructiva Crónica (EPOC) es una patología no transmisible, caracterizada por una limitación de flujo de aire en las vías respiratorias debido a una respuesta inmunológica anormal frente a partículas.

Objetivo. Conocer la eficacia que tiene la budesonida/formoterol comparado con la fluticasona/salmeterol en la mejoría de la capacidad pulmonar en personas mayores de 40 años con Enfermedad Pulmonar Obstructiva Crónica.

Materiales y métodos. Se realizó una revisión sistemática de documentos producidos entre el año 2000 y 2018 en distintas bases de datos, donde se incluyeron ensayos clínicos. Se identificaron cuatro artículos para el análisis final.

Resultados. Durante la evaluación comparativa de budesonida con formoterol, los artículos muestran un total de 709 personas evaluadas, con un promedio de edad de 53,5 años. El 65,4 \% eran varones, el $21 \%$ manifestaba no haber consumido tabaco, todos con diagnóstico de Enfermedad Pulmonar Obstructiva Crónica moderada-severa, según la escala GOLD (Global Initiative For Chronic Obstrutive Lung Disease). Los estudios determinaron que al administrar budesonida/formoterol de 400/12 mcg y 320/9 $\mathrm{mcg}$, los pacientes tuvieron una leve mejoría en el Volumen Espiratorio Forzado del primer segundo (VEF1). Solo dos pacientes presentaron efectos adversos. No obstante, para los resultados mencionados anteriormente no se encontró diferencias significativas. Conclusiones. El uso de budesonida/formoterol es eficaz al mejorar la capacidad ventilatoria pulmonar, disminuye el número de exacerbaciones anuales y genera un adecuado control de los síntomas, sin embargo, es igual de efectivo a la fluticasona/salmeterol. Palabras clave: Enfermedad Pulmonar Obstructiva Crónica, enfisema pulmonar, bronquitis crónica, formoterol, budesonida, salmeterol, fluticasona, eficacia.
\end{abstract}

\footnotetext{
${ }^{1}$ Facultad de Ciencias de la Salud, Universidad de Boyacá, Tunja, Colombia. ${ }^{2}$ Secretaría de Salud de Yopal, Yopal, Colombia

${ }^{3}$ Universidad de Antioquia, Medellin, Colombia. ${ }^{4}$ Centro de Rehabilitación Integral de Boyacá (CRIB). Tunja, Colombia Correspondencia: Ledmar J Vargas-Rodríguez Carrera 2a Este № 64-169 Tunja, Boyacá, Colombia Teléfono: 7450000 ext. 1104 Correo electrónico: lejovaro@gmail.com
}

Citar este artículo así: Vargas Rodríguez $\sqcup$, Solano Rojas FE, Díaz SL, Pinzón Olmos ML. Eficacia de la budesonida/formoterol comparado con la fluticasona/salmeterol en la mejoría de la capacidad pulmonar de personas mayores con Enfermedad Pulmonar Obstructiva Crónica: revisión sistemática de literatura.Revista Investig Salud Univ Boyacá. 2019;6(2): 53-70. doi: https://doi.org/10.24267/23897325.369 


\title{
Effectiveness of budesonide/formoterol compared to fluticasone / salmeterol in improving the lung capacity of older people with Chronic Obstructive Pulmonary Disease: systematic literature review
}

\begin{abstract}
Introduction. Chronic Obstructive Pulmonary Disease (COPD) is a not transmissible disease, characterized by a limitation of airflow in the respiratory tract, due to an abnormal immune response to particles.

Objective. This article aims to show that the application of budesonide / formoterol improves lung capacity in people over 40 years with Chronic Obstructive Pulmonary Disease.

Materials and methods. A systematic review was conducted in the period between 2000 and 2018 in different databases where clinical trials were included. Four articles were identified for the final analysis.

Results. During the comparative evaluation of budesonide with formoterol, a total of 709 people were evaluated, with an average age of 53.5 years, $65.4 \%$ were male, $21 \%$ reported not having used tobacco, all with a diagnosis of moderate-severe Chronic Obstructive Pulmonary Disease according to the GOLD scale (Global Initiative For Chronic Obstrutive Lung Disease). The studies determined that when budesonide / formoterol of 400/12 mcg and 320/9 mcg was administered, the patients had a slight improvement in the Forced Expiratory Volume of the first second (FEV1). Only two patients presented adverse effects. However, for the results mentioned above no significant differences were found.
\end{abstract}

Conclusions. The use of budesonide / formoterol is effective in improving pulmonary ventilatory capacity, decreases the number of annual exacerbations and generates adequate control of symptoms, however, it is equally effective in fluticasone / salmeterol.

Keywords: COPD; Chronic Obstructive Pulmonar Disease; pulmonary emphysema; chronic bronchitis; formeterol; budesonide; salmeterol; fluticasone; effectiveness. 


\section{Eficiência da budesonida/formoterol comparado com fluticasona/salmeterol na melhora da capacidade pulmonar de idosos com Doença Pulmonar Obstrutiva Crônica: revisão sistemática da literatura}

\section{RESUMO}

Introdução. A Doença Pulmonar Obstrutiva Crônica (DPOC) é uma patologia não transmissível, caraterizada por uma limitação do fluxo de ar nas vias aéreas devido a uma resposta imune anormal contra partículas. Objetivo. Conhecer a eficiência que apresenta a budesonida/formoterol comparado com fluticasona/salmeterol na melhora da capacidade pulmonar em pessoas com mais de 40 anos com Doença Pulmonar Obstrutiva Crônica.

Materiais e métodos. Foi realizada uma revisão sistemática dos documentos produzidos entre 2000 e 2018 em diferentes bancos de dados, onde foram incluídos ensaios clínicos. Quatro artigos foram identificados para a análise final.

Resultados. Durante a avaliação comparativa de budesonida com formoterol, os artículos mostram um total de 709 pessoas avaliadas, com uma idade média de 53,5 anos. 0 65,4 \% eram do sexo masculino, o 21 \% disseram que não usavam tabaco, todos diagnosticados com Doença Pulmonar Obstrutiva Crônica moderada a grave, de acordo com a escala GOLD (Global Initiative For Chronic Obstrutive Lung Disease). Os estudos determinaram que administrar budesonida/formoterol de $400 / 12 \mathrm{mcg}$ e $320 / 9$ mcg, os pacientes apresentaram uma leve melhora no Volume Expiratório Forçado no primeiro segundo (VEF1). Apenas dois pacientes tiveram efeitos adversos. No entanto, não foram encontradas diferenças significativas para os resultados mencionados acima.

Conclusões. O uso de budesonida/formoterol é eficaz na melhora da capacidade ventilatória pulmonar, diminui o numero de exacerbações anuais e gera controle adequado dos sintomas, no entanto, é igualmente eficaz para a fluticasona/salmeterol.

Palavras-chave: Doença Pulmonar Obstrutiva Crônica, enfisema, bronquite crônica, formoterol, budesonida, salmeterol, fluticasona, eficiência. 


\section{INTRODUCCIÓN}

La Enfermedad Pulmonar Obstructiva Crónica (EPOC) es una patología pulmonar, cuya característica principal es la limitación de flujo de aire en las vías respiratorias. Esta se asocia con una respuesta inmunológica anormal frente a partículas nocivas, además de lesión endotelial generalizada (1-6)controlled trials (RCTs; su presencia desencadena mecanismos de inflamación, fibrosis, destrucción de las pequeñas vías y la matriz proteica pulmonar, hipertrofia y aumento de secreción glandular, junto con mayor constricción del músculo liso bronquial $(7,8)$.

Esta entidad nosológica progresa de manera lenta y los primeros síntomas, generalmente, se presentan en la quinta década de la vida, caracterizados por disnea, tos y expectoración. La disnea es el síntoma de mayor relevancia clínica, pues empeora con la edad y se asocia con entornos de gran polución como los centros urbanos (4). Los síntomas producen incapacidad a la persona en actividades básicas de cuidado personal, debido a la falta de oxígeno y las infecciones recurrentes, e implican un mayor riesgo de sufrir cáncer y enfermedades cardiovasculares $(5,6,9)$ arterial hypertension, chronic heart failure. Sin embargo, en los casos leves se pueden o no presentar síntomas $(2,10,11)$.

De acuerdo con los datos reportados por la Organización Mundial de la Salud (OMS), la EPOC en 2016 tuvo una prevalencia de 251 millones de personas y representó el $5 \%$ de las muertes anuales. El 90 \% de ellas se dieron en países en vías de desarrollo (12).

La EPOC es una enfermedad compleja y está relacionada con múltiples factores de riesgo. Actualmente, se conocen alteraciones genéticas que establecen esta condición; sin embargo, son el ambiente, el entorno social y los hábitos de vida los que facilitan su desarrollo $(2,4,9,13)$. A la par de ello, se debe tener en cuenta que el ausentismo laboral del paciente y de los miembros de la familia relacionados con el cuidado del enfermo, implica una gran carga económica para los sistemas de salud, laboral y familiar, lo cual se suma a los años de vida potenciales perdidos $(5,6,14)$.

\section{Las guías Global Initiative For Chronic Obstrutive} Lung Disease (GOLD) han resumido las pautas del manejo de esta condición, por lo que se indica el uso de medicamentos para el alivio sintomático, el control de exacerbaciones y la mejoría en la calidad de vida $(13,15)$. Dentro de los medicamentos sugeridos como broncodilatadores están los beta agonistas, los cuales mejoran el Volumen Espiratorio Forzado del primer segundo (VEF1). Su acción principal es la relajación del músculo liso a través de la estimulación de los receptores adrenérgicos. La última generación de esta familia de medicamentos busca tener un mayor tiempo de acción con una vida media larga: Long 
Action Beta Agonist (LABA). Para el control de respuesta inflamatoria se asocia el beta agonista con un corticoide inhalado, cuya combinación da mejor resultado $(3,13,15)$.

Actualmente, el uso de corticoides más beta 2 adrenérgico de larga acción, está indicado en el tratamiento de personas con EPOC $(2,3,15,16)$. Por tal razón, este estudio tiene como objetivo establecer la eficacia de la budesonida/formoterol en la mejoría de la capacidad pulmonar de pacientes mayores de 40 años con EPOC (12).

\section{MATERIALES Y MÉTODOS}

Tipo de estudio: se realizó una revisión sistemática de literatura, esta revisión fue reportada de acuerdo con los lineamientos descritos en la declaración PRISMA 2.0 y en el Manual Cochrane de revisiones sistemáticas de intervenciones, versión 5.1.022. Además, fue registrada en la plataforma digital PROSPERO con el № 117049.

Población: pacientes mayores de 40 años con EPOC, que fueron tratados con formoterol/budesonida (intervención) en comparación con los tratados con salmeterol/fluticasona (comparación). De dichos medicamentos se evaluó, desde la literatura, la efectividad para mejorar la capacidad pulmonar mediante la VEF1, teniendo en cuenta algunos análisis secundarios como el número de exacerbaciones al año, los días de hospitalización y la mejoría de la calidad de vida en el paciente.

Criterios de inclusión: ensayos clínicos que evalúan la VEF1 de personas mayores de 40 años con EPOC, tratados con budesonida combinada con formeterol.

Criterios de exclusión: estudios que no comparan los medicamentos de interés en esta investigación y personas menores de 40 años. No se hicieron restricciones de sexo, raza o país. Se descartaron otros tipos de estudios como los estudios transversales, los reportes de caso, los estudios de series de casos, las revisiones de tema y las cartas editoriales.

Estrategia de búsqueda: las búsquedas fueron realizadas por dos investigadores, usando las bases de datos de Pubmed/Medline, Science direct, Scopus, Embase, Cochrane Library, BIREME, Redalyc, Scielo y el Directory of Open Acess Journals (DOAJ).

La estrategia de búsqueda utilizada fue:

COPD OR chronic obstructive pulmonar disease OR Chronic bronchitis OR pulmonary emphisema AND budesonide AND formeterol AND fluticasone AND salmeterol. 
Restricciones en la búsqueda: se limitó la búsqueda a la literatura relacionada con humanos mayores de 40 años y se redujo a los textos publicados en español e inglés.

Extracción de datos: estuvo a cargo de tres investigadores pertenecientes al proyecto, quienes como estrategia de búsqueda realizaron la identificación y la detección de la literatura a estudiar. Luego se seleccionaron los artículos según el título para elegir aquellos que fueran acordes al objetivo planteado. Posteriormente, se evaluaron los resúmenes de las investigaciones seleccionadas $y$, finalmente, se revisaron los artículos completos para ser elegidos e incluidos en el presente estudio. Las discrepancias que nacieron en el proceso se resolvieron por consenso y con la participación de un cuarto autor. Una vez elegidos los cuatro estudios aquí tratados, se extrajeron las características específicas de cada uno.

\section{Evaluación de la calidad metodológica: los estu-} dios incluidos no se encontraron en la plataforma PEDro, por eso, estos fueron evaluados por cada uno de los investigadores (Tabla 1), obteniendo un solo resultado que evalúa el porcentaje de cumplimiento de cada ítem.

Tabla 1. Evaluación metodológica de los estudios mediante PEDro.

\begin{tabular}{ccccccccccccc}
\hline & Referencia & P1 & P2 & P3 & P4 & P5 & P6 & P7 & P8 & P9 & P10 & Total \\
\hline $\begin{array}{c}\text { Cazzola 2003 } \\
\text { (10) }\end{array}$ & + & - & + & + & - & - & + & + & + & + & 70 \\
\hline & $\begin{array}{c}\text { Dalby 2009 } \\
(11)\end{array}$ & + & + & - & + & - & - & + & + & + & + & 70 \\
\hline & $\begin{array}{c}\text { Patridge 2009 } \\
(12)\end{array}$ & + & - & + & + & + & - & + & + & + & + & 80 \\
\hline & $\begin{array}{c}\text { Linberg 2007 } \\
(13)\end{array}$ & + & + & + & + & + & + & - & + & + & + & 90 \\
\hline & $\begin{array}{c}\text { Cumplimiento } \\
(\%)\end{array}$ & 100 & 50 & 75 & 100 & 50 & 25 & 75 & 100 & 100 & 100 & 77,5 \\
\hline
\end{tabular}

P1: Criterios de elección mencionados. P2: Asignación al azar de los grupos. P3: Asignación oculta. P4: Los grupos fueron similares. P5: Cegamiento. P6: Cegamiento de los investigadores. P7: Las medidas de al menos uno de los resultados clave fueron obtenidas de más del 85 \% de los sujetos inicialmente asignados a los grupos. P8: Se presentaron resultados de todos los sujetos que recibieron tratamiento. P9: Los resultados de comparaciones estadísticas entre grupos fueron informados para al menos un resultado clave. P10: El estudio proporciona medidas puntuales y de variabilidad para al menos un resultado clave.

(+): Cumplió. (-): No cumplió. 
Posteriormente, se calculó el índice de Kappa para determinar la concordancia en la evaluación de los distintos observadores (Tabla 2). Esta escala propuesta por Cohen en 1960 (17) excluye la concordancia atribuible al azar, con el fin de reflejar la concordancia genuina de dos o más observaciones. Considera como estudios aceptables los que muestran concordancia mayor a 0,4 , y excelentes aquellos que superan 0,75 . Los datos que están por debajo de lo planteado se consideran no pertinentes para el estudio por baja calidad (17-19).

Tabla 2. Concordancia de Kappa de Cohen aplicada a los estudios seleccionados para la investigación.

\begin{tabular}{ccc}
\hline Artículo & $\begin{array}{c}\text { Índice de Kappa } \\
\text { de Cohen }\end{array}$ & Concordancia \\
\hline Cazzola 2003 (10) & 0.85 & Excelente \\
\hline Dalby 2009 (11) & 0.72 & Buena \\
\hline Patridge 2009 (12) & 0.52 & Aceptable \\
\hline Linberg 2007 (13) & 0.63 & Buena \\
\hline
\end{tabular}

\section{Riesgo de sesgo}

Basados en los lineamientos del Manual Cochrane de revisiones sistemáticas, se evaluaron los tipos de sesgo de cada uno de los estudios, así:
- Sesgo de selección: Los autores describieron el método utilizado para generar la secuencia de asignación y la ocultaron.

- Sesgo de realización: los investigadores mencionaron todas las medidas utilizadas, para así cegar a los participantes y al personal del estudio sobre las intervenciones aplicadas.

- Sesgo de detección: dentro del manuscrito se proporcionó información acerca de los métodos utilizados, para cegar a los evaluadores del resultado del estudio.

- Sesgo de desgaste: se señaló la compleción de los datos de resultado para cada resultado principal, incluidos los abandonos, las exclusiones del análisis y los motivos de cada uno de ellos.

- Sesgo de notificación: se observó selectividad para el reporte y la notificación de los resultados.

- Otros sesgos: sesgos no abordados en los apartados anteriores.

A partir de el aplicativo REVMAN, se resumió el análisis de los posibles sesgos de las investigaciones. A partir de él también se generó gráficamente un resumen de los riesgos (Figura 1). 
Figura 1. Riesgo de sesgo de cada uno de los estudios encontrados.

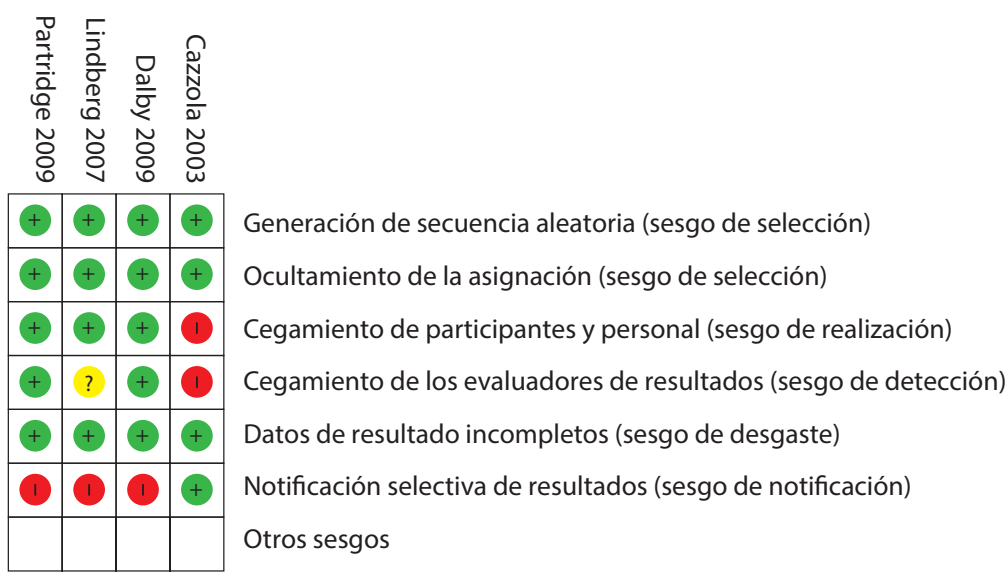

\section{RESULTADOS}

\section{Selección de estudios}

De acuerdo con los criterios establecidos y la estrategia de búsqueda, la exploración arrojó un total de 120 artículos. Se excluyeron 112 investigaciones tras la aplicación de los límites y luego se eliminaron 5 artículos por considerarlos no pertinentes para la resolución de la pregunta de investigación. Finalmente, se hizo la revisión de los artículos completos y se retiró un artículo al encontrarse repetido. De esta manera, se consideraron solamente cuatro estudios como pertinentes para dar respuesta a la pregunta de investigación. El proceso de selección de dichos estudios se muestra a través del diagrama de flujo (Figura 2), de acuerdo con la declaración de PRISMA.

\section{Características de los estudios}

Se incluyeron un total de cuatro ensayos clínicos realizados entre los años 2003 y 2018 en países norteamericanos, en donde se compara salmeterol/fluticasona con formoterol/budesonida (1013). Dos de ellos son ensayos cruzados $(10,13)$ y otros dos, paralelos $(11,12)$.

\section{Características de la población}

Un total de 709 personas fueron incluidas. De ellas, el 65,4 \% eran varones (464) y el 34,6\%, mujeres. El promedio de edad de la población incluida fue de 53,5 años (DE 11,9). La totalidad de la población tenía diagnóstico previo de Enfermedad Pulmonar Obstructiva Crónica, clasificada mediante escala GOLD como moderada a severa, 
Figura 2. Diagrama de búsqueda y selección de los estudios

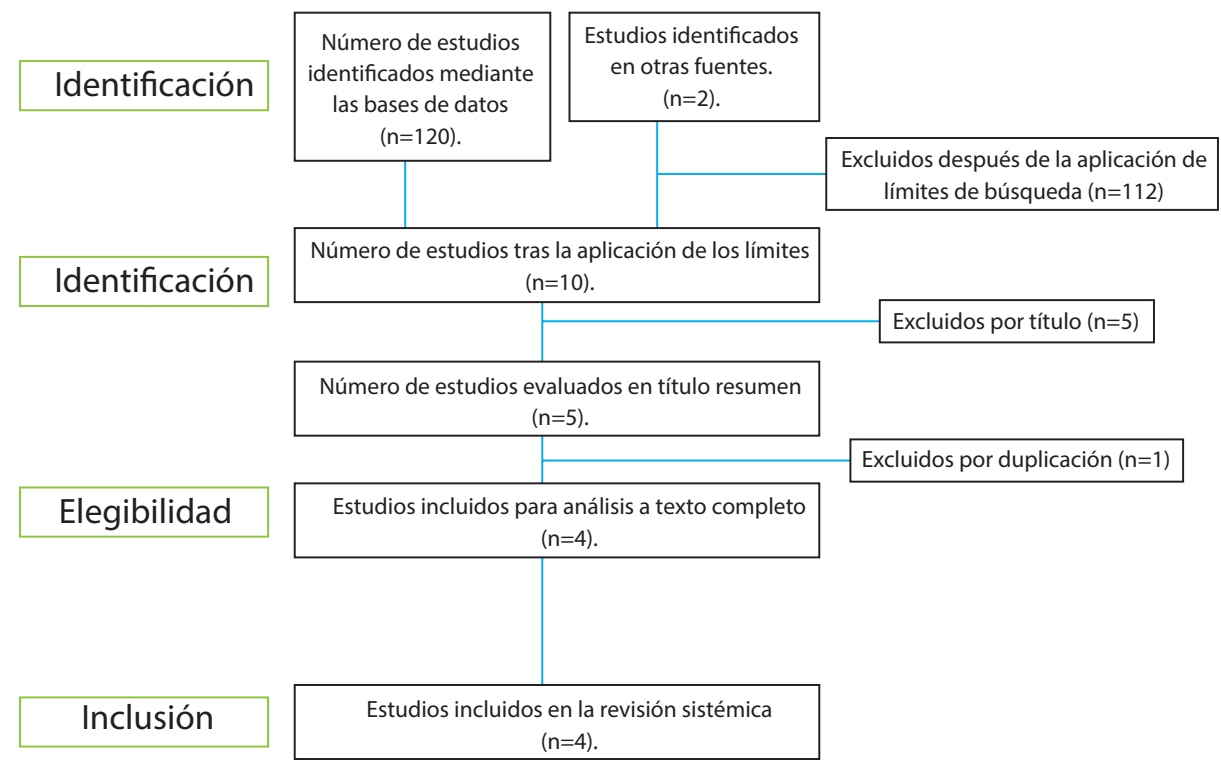

con una VEF1 basal promedio general de 49,7 \%. Del total de participantes, 311 personas eran fumadoras, 249 exfumadoras y 149 no habían fumado.

\section{Características de las intervenciones}

Las dosis de budesonida/formoterol fueron de $400 / 12 \mathrm{mcg}(10,12), 320 / 9 \mathrm{mcg}(11)$ y $160 / 4,5$ mcg (13), tal como vienen en las distintas presentaciones. Se administraron en su totalidad dos puff al día y se evaluó la función ventilatoria momentos después de su administración, con el fin de observar si generaba o no cambios en la población.
Sin embargo, algunos lo administraron como una única dosis y después de un tiempo de limpieza realizaron cambio de medicamento, evaluando lo mismo. En otros estudios, las dosis se mantuvieron por tres semanas a medida que se adelantaba la evaluación del paciente.

\section{Características de la comparación}

Los estudios manejaron dosis distintas. Para el caso de salmeterol/fluticasona de 50/250 y $50 / 500$ microgramos, estos fueron administrados mediante inhalador de dosis medida, tal como vienen en su presentación. 
Se administraban en su totalidad dos puff al día y se evaluaba la función ventilatoria momentos después de su administración, con el fin de verificar si generaba o no cambios en la población.

Sin embargo, similar a las intervenciones, algunos lo administraron como una única dosis y después de un tiempo de limpieza realizaron cambio de medicamento, evaluando lo mismo. En otros estudios las dosis se mantuvieron por tres semanas a medida que avanzaba la evaluación del paciente.

\section{Medidas de resultado primarias}

Se analizaron los medicamentos según las dosis administradas: dos estudios manejaron dosis de budesonida/formoterol de 400/12 microgramos $(10,12)$, los cuales iniciaban con una base y aumentaban el VEF1 a los 120 minutos, en un promedio de 0,95\%; a los 300 minutos, en 1,1\%; y a los 720 minutos aumentaba $0,4 \%$. Sin embargo, al comparar esta variación con el grupo control, no tiene significancia estadística y los valores son similares al otro medicamento utilizado (Tabla 3).

En el estudio donde se manejaron dosis de 320/9 microgramos (11) se encontró que aumentaba el VEF1 a los 120 minutos en un promedio de $1,5 \%$; a los 300 minutos en $0,5 \%$; y a los 720 minutos aumentaba $1 \%$. Sin embargo, al igual que en la variación anterior, compararla con el grupo control no tiene significancia estadística y los valores son similares al otro medicamento utilizado (Tabla 3).

En el estudio donde se manejaron dosis de 160/4,5 microgramos (13) se encontró que aumentaba el VEF1 a los 120 minutos en un promedio de 3,8 $\%$; a los 300 minutos en $2 \%$; y a los 720 minutos aumentaba $0,1 \%$. Sin embargo, una vez más, al comparar esta variación con el grupo control, no tiene significancia estadística y los valores son similares al otro medicamento utilizado (Tabla 3).

Estos resultados demuestran que los pacientes tratados con esta combinación de medicamentos tienen poca respuesta broncodilatadora $y$, al compararlos con otros medicamentos de las mismas características, no presentan diferencias significativas. Además, se observó que las dosis más bajas generan en los pacientes un aumento mayor en el Volumen Espiratorio Forzado en el primer segundo.

\section{Medidas de resultados secundarios}

Las investigaciones mostraron que el número de exacerbaciones de EPOC al año en el grupo manejado con budesonida/formoterol fue de 0,80 y en el grupo control fue de 1,09. Esto indica un $26,6 \%$ menos en el grupo experimento que en el grupo control, con significancia estadística $(p=<0,005)(11,14)$. Además, refieren que este medicamento disminuye el tiempo de estancia hospitalaria, pues tiene un índice de 0,15 días 
menos de hospitalización, comparado con 0,21 en el grupo control. Esto indica una diferencia del $29,1 \%$ entre los grupos $(11,14)$.

Adicionalmente, solo un estudio evaluó la percepción de mejoría de la calidad de vida y de control de síntomas mediante la pregunta: ¿Sintió mejoría luego de administrado el tratamiento? A partir de ello se observó que los pacientes manejados con budesonida/formoterol tuvieron respuesta favorable en un $84 \%$, en comparación del $81 \%$ del grupo control. Sin embargo, esto no generó diferencia estadística (12).

En otro estudio, se evaluó la cantidad de expectoración del paciente luego de administrado el medicamento (corticoide) y se observó que el uso de fluticasona disminuye el volumen en un $15 \%$ $(13,14)$.

Efectos adversos: en total, dos pacientes presentaron efectos adversos, uno presentó exacerbación y otra nasofaringitis.

\section{DISCUSIÓN}

La EPOC es una enfermedad de importante impacto en la funcionalidad de la población adulta mayor, en el contexto mundial y regional, con gran efecto en la percepción de calidad de vida y en el pronóstico de otras patologías crónicas $(5,6,9,13)$. Su tratamiento propone mejorar la función ventilatoria a través de la broncodilatación. La combinación de un beta agonista y un corticoide inhalados ha mostrado la mayor efectividad hasta el momento $(2,3,16)$.

Las dos combinaciones más usadas de esta medicación son budesonida/formeterol y salmeterol/ fluticasona $(3,13,15)$. Las investigaciones incluidas en esta revisión sistemática compararon la efectividad de estos tratamientos con respecto a la función pulmonar, la percepción de mejoría y la funcionalidad en actividades básicas (20-23).

La evaluación realizada por los investigadores reportó niveles adecuados de aplicación metodológica en los estudios incluidos, con concordancia apropiada según el índice Kappa, que varió entre 0,52 y 0,85 (17-19). Adicionalmente, el riesgo de sesgo en las investigaciones fue bajo, a excepción del posible sesgo de publicación que algunos artículos no aclaran explícitamente, sin que esto signifique pérdida de validez en sus resultados.

Al condensar la información se encontró una población total de 709 participantes, en su mayoría hombres, con promedio de edad de 53 años y con diagnóstico de EPOC clasificado GOLD moderado o severo (13). La única excepción fueron los 27 participantes sanos de Dalby en 2009.

Se analizó la budesonida/formeterol como intervención y fluticasona/salmeterol como comparación. Se 
consideraron como resultados primarios los cambios en la función pulmonar VEF1 y como resultados secundarios las exacerbaciones anuales, la estancia hospitalaria y la percepción de calidad de vida (2).

Como hallazgos se encontró que los pacientes que usan budesonida/formoterol tienen mejoría en la capacidad pulmonar (10-13). También, los resultados obtenidos demostraron que el uso de esta combinación tiene mejor consecuencia a corto y mediano plazo que el salmeterol/fluticasona, pues la percepción que tuvieron los pacientes sobre su mejoría fue un efecto más rápido y duradero (20-22).

Una de las investigaciones comparó las concentraciones séricas y en esputo del medicamento, y reportó que la budesonida/formeterol es ligeramente más duradera en sangre que el salmeterol/fluticasona (23). Además, se muestra mejoría en el cuadro clínico y en la calidad de vida del paciente con EPOC (20-22), y una diferencia en la cuantificación de la VEF1 y la percepción de mejoría, mayor con budesonida/formeterol en estas investigaciones $(20,21)$.

En general, el mayor cambio en la función pulmonar se da a 100 minutos de la dosis de inhalador (20). Todos los estudios concluyeron que a los 720 minutos de la dosis se da la mejor condición pulmonar respecto a la medición basal, pero sin diferencia relevante con la misma (20-22).
El aumento en la frecuencia cardiaca y los cambios en la saturación arterial de oxígeno fueron mediciones adicionales entre las que no se encontró diferencia significativa, aunque el pico de frecuencia cardiaca se dio a los $\mathbf{3 0 0}$ minutos para ambas medicaciones, posiblemente asociado a comorbilidades propias de este tipo de pacientes $(5,9,20)$.

En cuanto a los resultados secundarios, se encontró que el número de exacerbaciones al año, en el grupo manejado con budesonida/formoterol, fue de 0,80 y el grupo control fue de 1,09. Esto indica un $26,6 \%$ menos de probabilidad de esta complicación, por lo que además, se disminuye el número de días de hospitalización (2, 20-23).

Si bien se nota mayor efectividad en el manejo de la EPOC por budesonida/formeterol que por salmeterol/fluticasona, esta diferencia es discreta y no tiene significancia estadística en la mayoría de las variables estudiadas. Aún es discusión cuál de estas opciones aplicar para cada caso en particular, siendo el concepto clínico del tratante el que define finalmente; sin embargo, se presenta un escenario prometedor en prevención y tratamiento de la EPOC, así como en el control de la limitación en la funcionalidad de quienes la padecen $(3,15)$. 
Tabla 3. VEF1 en pacientes con EPOC analizados.

\begin{tabular}{|c|c|c|c|c|c|c|c|c|c|c|c|c|c|c|c|c|}
\hline \multirow[b]{2}{*}{ AUTOR, AÑO } & \multirow[b]{2}{*}{ DISEÑO } & \multicolumn{4}{|c|}{ POBLACIÓN } & \multicolumn{3}{|c|}{ TABAQUISMO } & \multirow[b]{2}{*}{$\begin{array}{l}\text { SEVERIDAD } \\
\text { DE EPOC }\end{array}$} & \multirow[b]{2}{*}{$\begin{array}{l}\text { MEDICAMENTO } \\
\text { (PRESENTACIÓN) }\end{array}$} & \multirow[b]{2}{*}{$\begin{array}{l}\text { FRECUENCIA DE } \\
\text { ADMINISTRACIÓN }\end{array}$} & \multirow[b]{2}{*}{ DURACIÓN } & \multicolumn{4}{|c|}{ RESULTADO } \\
\hline & & TOTAL & & $\mathrm{H} / \mathrm{F}$ & $\begin{array}{l}\text { PROMEDIO } \\
\text { DE EDAD } \\
\text { (ANNOS) } \\
\end{array}$ & sí & No & $\mathrm{EX}$ & & & & & $\begin{array}{l}\text { VEF1 BASAL } \\
\text { PROMEDIO }\end{array}$ & $\begin{array}{l}\text { VEF1 } 120 \\
\text { MIN }\end{array}$ & $\begin{array}{l}\text { VEF1 } 300 \\
\text { MIN }\end{array}$ & $\begin{array}{l}\text { VEF1 } 720 \\
\text { MIN }\end{array}$ \\
\hline \multirow{2}{*}{$\begin{array}{l}\text { Cazzola, } 2003 \\
\text { (10) }\end{array}$} & \multirow{2}{*}{ EC CRUZADO } & GRUPO EXPERIMENTO & 16 & $14 / 2$ & 65 & 5 & 0 & 11 & $\begin{array}{l}\text { Moderado } \\
\text { severo }\end{array}$ & $\begin{array}{l}\text { Budesonida/formoterol } \\
400 / 12 \mu \mathrm{g}\end{array}$ & 2 PUFF AL DÍA & 2 DíAS & $47 \%$ & $47,90 \%$ & $48 \%$ & $48,90 \%$ \\
\hline & & GRUPO CONTROL & 16 & $14 / 2$ & 65 & 5 & 0 & 11 & $\begin{array}{l}\text { Moderado } \\
\text { severo }\end{array}$ & 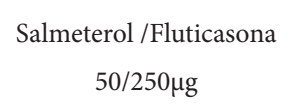 & 2 PUFF AL DÍA & 2 DíAS & $47 \%$ & $47 \%$ & $48,50 \%$ & $48,20 \%$ \\
\hline \multirow{2}{*}{$\begin{array}{l}\text { Patridge, } 2009 \\
\quad(12)\end{array}$} & \multirow{2}{*}{$\begin{array}{l}\text { EC DOBLE CIEGO, } \\
\text { MULTICENTRICO, } \\
\text { CUZADO }\end{array}$} & GRUPO EXPERIMENTO & 217 & $152 / 65$ & 62 & 75 & 10 & 132 & $\begin{array}{l}\text { Moderado } \\
\text { severo }\end{array}$ & $\begin{array}{c}\text { Budesónida/formeterol } \\
320 / 9 \mu g\end{array}$ & 2 PUFF AL DÍA & 2 SEMANAS & $60 \%$ & $61,50 \%$ & $62 \%$ & $63 \%$ \\
\hline & & GRUPO CONTROL & 225 & $167 / 58$ & 59 & 82 & 15 & 128 & $\begin{array}{l}\text { Moderado } \\
\text { severo }\end{array}$ & $\begin{array}{l}\text { Salmeterol/fluticasona } \\
50 / 500 \mu \mathrm{g}\end{array}$ & DOS PUFF AL DÍA & 3 SEMANAS & $58 \%$ & $58,50 \%$ & $60 \%$ & $61 \%$ \\
\hline \multirow{2}{*}{$\begin{array}{l}\text { Dalby, } 2009 \\
\text { (11) }\end{array}$} & \multirow{2}{*}{$\begin{array}{c}\text { EC MULTICÉNTRI- } \\
\text { CO DOBLE CIEGO } \\
\text { DOBLE DUMMY } \\
\text { CRUZADO }\end{array}$} & GRUPO EXPERIMENTO & 28 & $21 / 7$ & 65 & 22 & 0 & 16 & $\begin{array}{l}\text { Moderado } \\
\text { severo }\end{array}$ & $\begin{array}{l}\text { Budesonida/formoterol } \\
400 / 12 \mu \mathrm{g} \text { + placebo }\end{array}$ & DOS PUFF AL DÍA & ÚNICA & $42 \%$ & $43 \%$ & $43,20 \%$ & $43,20 \%$ \\
\hline & & GRUPO CONTROL & 27 & $11 / 16$ & 52 & 0 & 27 & 0 & Sano & 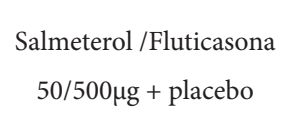 & DOS PUFF AL DÍA & ÚNICA & $82 \%$ & $84 \%$ & $84,20 \%$ & $85,00 \%$ \\
\hline \multirow{2}{*}{$\begin{array}{l}\text { Lindberg, } 2009 \\
\quad(13)\end{array}$} & \multirow{2}{*}{ EC CRUZADO } & GRUPO EXPERIMENTO & 89 & $50 / 39$ & 62 & 54 & 0 & 32 & $\begin{array}{l}\text { Moderado } \\
\text { severo }\end{array}$ & $\begin{array}{l}\text { Budesónida/formeterol } \\
\quad 160 / 4.5 \mu \mathrm{g}\end{array}$ & DOS PUFF AL DÍA & ÚNICA & $60,2 \%$ & $64 \%$ & $66 \%$ & $66,1 \%$ \\
\hline & & GRUPO CONTROL & 89 & $50 / 39$ & 62 & 54 & 0 & 32 & $\begin{array}{l}\text { Moderado } \\
\text { severo }\end{array}$ & $\begin{array}{l}\text { Salmeterol /Fluticasona } \\
50 / 250 \mathrm{\mu g}\end{array}$ & DOS PUFF AL DÍA & ÚNICA & $60,2 \%$ & $63 \%$ & $64,2 \%$ & $64,3 \%$ \\
\hline
\end{tabular}

H: Masculino F: Femenino. EC: Ensayo Clínico. EX: Exfumador. EPOC: Enfermedad Pulmonar Obstructiva Crónica. VEF1: Volumen Espiratorio Forzado el primer segundo. 


\section{CONCLUSIÓN}

La combinación de budesonida con formoterol muestra beneficios para los pacientes que la usan, puesto que mejora la capacidad pulmonar, sin que tengan significancia estadística. El mejoramiento es más rápido y duradero con la combinación formeterol/budesonida que con salmeterol/fluticasona y es por criterio clínico que debe seleccionarse una de las dos combinaciones, según las particularidades de cada caso.

A futuro, se requieren más investigaciones que evalúen el número de exacerbaciones y la duración intrahospitalaria.

\section{RESPONSABILIDADES ÉTICAS}

Dentro de los estudios analizados se observan los debidos lineamientos éticos y bioéticos para el ingreso, permanencia y retiro del estudio. Se considera que dichos estudios, y este en particular, cumplen con los principios éticos de investigaciones médicas en seres humanos de acuerdo con la declaración de Helsinki (20-24).

\section{FINANCIACIÓN}

Esta investigación fue financiada con recursos de los autores y recibió apoyo económico de la Universidad de Boyacá.

\section{CONFLICTOS DE INTERÉS}

Los autores no refieren conflictos de interés.

\section{REFERENCIAS}

1. Wilt TJ, Niewoehner D, MacDonald R, Kane RL. Management of stable chronic obstructive pulmonary disease: a systematic review for a clinical practice guideline. Ann Intern Med [Internet]. 2007 Nov 6 [cited 2019 Mar 11];147(9):639-53. Disponible en: https://doi. org/10.7326/0003-4819-147-9-20071106000009

2. Brett AS, Al-Hasan MN. COPD Exacerbations - A Target for Antibiotic Stewardship. N Engl J Med [Internet]. 2019 Jul 11 [cited 2019 Jul 18];381(2):174-5. Disponible en: https://doi. org/10.1056/NEJMe1905520

3. Soriano JB. The evolution of COPD species; or, something is changing for good in COPD. Eur Respir J [Internet]. 2019 Jun 27 [cited 2019 Jul 18];53(6):1900762. Disponible en: https://doi. org/10.1183/13993003.00762-2019.

4. Zhang L, Feng $W$, Lu BB, Li N, Li H, Gu SH, et al. [The impact of ambient $\operatorname{PM}(2.5)$ on daily outpatient visits due to chronic obstructive pulmonary disease, among the urban residents of Ningbo city]. Zhonghua Liu 
Xing Bing Xue Za Zhi [Internet]. 2019 Jun 10 [cited 2019 Jul 18];40(6):686-91. Disponible en: https://doi.org/10.3760/cma.j.is sn.0254-6450.2019.06.016

5. Karoli NA, Rebrov AP. Endothelial dysfunction in patients with chronic obstructive pulmonary disease in combination with coronary heart disease. Ter Arkh [Internet]. 2019 Mar 29 [cited 2019 Jul 18];91(3):22-6. Disponible en: https://doi.org/10.26442/00403660.2019. 03.000061

6. Grigoryeva NY, Maiorova MV, Korolyova ME, Samolyuk MO. Comorbidity and polymorbidity of the patient with chronic obstructive pulmonary disease and cardiovascular diseases. Ter Arkh [Internet]. 2019 Mar 10 [cited 2019 Jul 18];91(1):38-47. Disponible en: https://doi. org/10.26442/00403660.2019.01.000027

7. Hurst JR, Vestbo J, Anzueto A, Locantore N, Müllerova $\mathrm{H}$, Tal-Singer $\mathrm{R}$, et al. Susceptibility to Exacerbation in Chronic Obstructive Pulmonary Disease. N Engl J Med [Internet]. 2010 Sep 16 [cited 2019 Mar 11];363(12):112838. Disponible en: https://doi.org/10.1056/ NEJMoa0909883

8. Vestbo J, Hurd SS, Agustí AG, Jones PW, Vogelmeier $C$, Anzueto A, et al. Global Strategy for the Diagnosis, Management, and Prevention of Chronic Obstructive Pulmonary Disease. Am J Respir Crit Care Med [Internet]. 2013 Feb 15 [cited 2019 Mar 11];187(4):347-65. Disponible en: https://doi.org/10.1164/rccm.201204-0596PP

9. Belenkov YN, Tsvetkova OA, Privalova E V., An G V., Ilgisonis IS, Voronkova OO. Comorbidity of Chronic Obstructive Pulmonary Disease and Cardiovascular Diseases: Place of Therapy with Modern $\beta$-Adrenoblockers. Kardiologiia [Internet]. 2019 Jun 27 [cited 2019 Jul 18];59(6):4855. Disponible en: https://doi.org/10.18087/ cardio.2019.6.n458

10. Peces-Barba G, Barberà JA, Agustí A, Casanova $C$, Casas $A$, Izquierdo JL, et al. [Diagnosis and management of chronic obstructive pulmonary disease: joint guidelines of the Spanish Society of Pulmonology and Thoracic Surgery (SEPAR) and the Latin American Thoracic Society (ALAT)]. Arch Bronconeumol [Internet]. 2008 May [cited 2019 Mar 11];44(5):271-81. Disponible en: http://www. ncbi.nlm.nih.gov/pubmed/18448019

11. Holleman DR, Simel DL, Goldberg JS. Diagnosis of obstructive airways disease from the clinical examination. J Gen Intern Med [Internet]. 1993 Feb [cited 2019 Mar 12];8(2):63-8. Disponible en: https://doi.org/10.1007/BF02599985 
12. Mathers CD, Loncar D. Projections of Global Mortality and Burden of Disease from 2002 to 2030. Samet J, editor. PLoS Med [Internet]. 2006 Nov 28 [cited 2019 Mar 12];3(11):e442. Disponible en: https://doi.org/10.1371/journal. pmed.0030442

13. Mirza S, Clay RD, Koslow MA, Scanlon PD. COPD Guidelines: A Review of the 2018 GOLD Report. Mayo Clin Proc [Internet]. 2018 Oct 1 [cited 2019 Mar 12];93(10):1488-502. Disponible en: https://doi.org/10.1016/j. mayocp.2018.05.026

14. Gibson GJ, Loddenkemper $R$, Lundbäck $B$, Sibille $Y$. Respiratory health and disease in Europe: the new European Lung White Book. Eur Respir J [Internet]. 2013 Sep 2 [cited 2019 Mar 12];42(3):559-63. Disponible en: https:// doi.org/10.1183/09031936.00105513

15. Avdeev SN, Trushenko NV. New opportunities of dual bronchodilation therapy for patients with chronic obstructive pulmonary disease. Ter Arkh [Internet]. 2019 Mar 30 [cited 2019 Jul 18];91(3):76-85. Disponible en: https://doi.org/ 10.26442/00403660.2019.03.000136

16. Kew KM, Dias $\mathrm{S}$, Cates CJ. Long-acting inhaled therapy (beta-agonists, anticholinergics and steroids) for COPD: a network meta-analysis. Cochrane Database Syst Rev
[Internet]. 2014 Mar 26 [cited 2019 Mar 12];(3):CD010844. Disponible en: https://doi. org/10.1002/14651858.CD010844.pub2

17. Cohen J. A Coefficient of Agreement for Nominal Scales. Educ Psychol Meas [Internet]. 1960 Apr 2 [cited 2019 Jul 18];20(1):37-46. Disponible en: https://doi. org/10.1177/001316446002000104

18. Campo-Arias A, Herazo E. Concordancia intra-e interevaluadores. Rev Colomb Psiquiat [Internet]. 2010 [cited 2019 Jul 18];39(2):42332. Disponible en: http://www.redalyc.org/ pdf/806/80615447015.pdf

19. Tang W, Hu J, Zhang H, Wu P, He H. Kappa coefficient: a popular measure of rater agreement. Shanghai Arch Psychiatry. 2015;27(1):62-7. https://doi.org/10.11919/j. issn.1002-0829.215010

20. Cazzola M, Santus P, Di Marco F, Boveri B, Castagna $F$, Carlucci $P$, et al. Bronchodilator effect of an inhaled combination therapy with salmeterol + fluticasone and formoterol + budesonide in patients with COPD. Respir Med [Internet]. 2003 May [cited 2019 Jun 30];97(5):453-7. Disponible en: https://doi. org/10.1053/rmed.2002.1455 
21. Lindberg A, Szalai Z, Pullerits T, Radeczky E. Fast onset of effect of budesonide/formoterol versus salmeterol/fluticasone and salbutamol in patients with chronic obstructive pulmonary disease and reversible airway obstruction. Respirology [Internet]. 2007 Sep [cited 2019 Jun 30];12(5):732-9. Disponible en: https://doi.org/10.1111/j.1440-1843.2007.01132.x

22. Partridge $M R$, Schuermann $W$, Beckman O, Persson T, Polanowski T. Effect on lung function and morning activities of budesonide/formoterol versus salmeterol/fluticasone in patients with COPD. Ther Adv Respir Dis [Internet]. 2009 Aug 4 [cited 2019 Jun 30];3(4):147-57. Disponible en: https://doi. org/10.1177/1753465809344870

23. Dalby C, Polanowski T, Larsson T, Borgström L, Edsbäcker S, Harrison TW. The bioavailability and airway clearance of the steroid component of budesonide/formoterol and salmeterol/fluticasone after inhaled administration in patients with COPD and healthy subjects: a randomized controlled trial. Respir Res [Internet]. 2009 Oct 31 [cited 2019 Jul 1];10(1):104. Disponible en: https://doi.org/10.1186/14659921-10-104

24. World Health Organization. WMA Declaration of Helsinki - Ethical Principles for Medical Research Involving Human Subjects - WMA -
The World Medical Association [Internet]. Helsinky; 2013 [cited 2019 Jul 18]. Disponible en: https://www.wma.net/policies-post/wma-declaration-of-helsinki-ethical-principles-for-medical-research-involving-human-subjects/

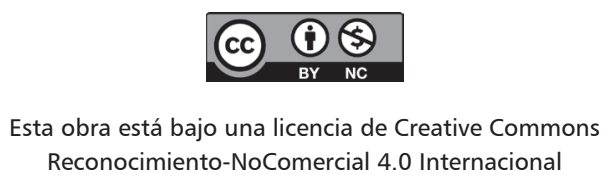

sta obra está bajo una licencia de Creative Commons 